Editorial

\title{
Editors and Journal Startup in the Digital Era
}

\author{
Andrew Kirby \\ School of Social and Behavioral Sciences, Arizona State University, Phoenix, AZ 85069-7100, USA; \\ E-Mail: andrew.kirby@asu.edu
}

Received: 24 September 2015 / Accepted: 29 September 2015 / Published: 12 October 2015

\section{Introduction}

More has changed in journal publishing in the past twenty years than the previous four centuries. Digital technologies have transformed the submission, review, production and distribution of scholarly materials, with the result that there has been exponential growth in the number of papers published in an expanding roster of journals — some are mainstream, some highly specialized, some are produced by publishers who have existed since printing began and others are produced by small groups with niche interests.

With these changes as the backdrop, this special issue was designed to collect papers from those with recent professional experience in the production of scholarly journals. Invitations were extended to editors and editorial board members who are working to create new journals or who have addressed the challenges of maintaining established journals in a crowded marketplace.

Several specific questions were crafted to guide participants.

1. What was the stated purpose of the journal? Was it to address a new scientific reality, a new empirical reality, a new policy environment? Is the journal generating a new readership; perhaps a new authorship? Is it meeting the expectations that drove the journal's creation?

2. To address that latter question: what metrics are important to the journal's editors and Board? Traditional ones (Impact Factors); more market oriented measures, such as downloads? Does the Board care about traditional citations in Web of Science or does it see suitable breadth in Google Scholar?

3. What is the organizational structure of the journal: does it possess multiple editors? How large is the Board? What determined the structure that has emerged: is it designed for geographical coverage, a form of diversity, or does it reflect scientific organization?

4. What role has open access (OA) played in the creation and subsequent operation of the journal? Was it a key factor or a minor aspect as far as editors are concerned? Is it important to authors, as far as one can tell? 
5. What about reviews: is it proving hard to obtain quality reviews? Does the journal face issues relating to authors from emerging nations? Copyright and integrity issues?

6. Are production issues of importance: do authors care about the appearance of their manuscripts in this era of blogs and extensive pre-publication?

\section{The Special Issue}

The formal call for papers did not generate any responses and so manuscripts were solicited from diverse contacts, including editors producing journals for the Multidisciplinary Digital Publishing Institute (MDPI, Basel, Switzerland), editors producing social science journals for Elsevier (Amsterdam, The Netherlands), and personal contacts of the author.

Eventually, nine participants agreed to submit a paper dealing with their experiences as an editor as this relates to the goals of the special issue. This group contained a balance of social science and humanities representatives, and journals produced by commercial publishers, university presses, and universities.

Unfortunately, there was subsequently a high rate of attrition, reducing the collection to the five papers that follow, plus an article by myself [1]. All are from editors working in the social sciences and all edit a journal produced by Elsevier.

\section{Assessment}

The papers all provide interesting insights into the role of an editor working within the STEM network of larger corporations such as Elsevier, Springer (Berlin, Germany), Wiley (Hoboken, NJ, USA), or Taylor and Francis (London, UK). Steinberg [2] in fact structures his statement on the manner in which these corporate publishers have expanded their reach in recent years, removing for example the opportunity to publish "directly" with learned societies, whose journals are for the most part now managed by for-profit publishers with an eye on increasing revenue Steinberg [2].

Two papers deal with transport, a subject area in which there is ever greater specialization. This reflects both the importance of transport within a connected global economy, and the complexity of travel modes. Both these papers can be parsed for insights into the working life of the journal editor, attempting to build up a readership and to publish high quality manuscripts. The burdens of being continually "in touch" via an online system, through which authors and reviewers route their various materials, are clear. That said, the strategies of the editors are very different. Mindell [3] writes about the development of a journal supporting an emerging multidisciplinary field, and offers a concise summary of the statistics associated with the journal; especially impressive is the fact that the journal has been indexed after only one year of operation, a key metric for the solicitation of further high quality manuscripts.

In contrast, Brooks and Ison [4] have opted for a strategy of assembling special issues, which has great potential payoff (what they term "the blessing") but is also significantly more labor-intensive ("the curse"). Their discussion of this route to growth also raises one of the significant questions about the digital market place, namely the existence of cumulative value in the scholarly journal (Phillips [5]). Decades ago, as print journals increased in number, they did so because they provided a service to a specific readership (for instance, the members of a learned society). Book reviews indicated what 
was being written about within a field and who was writing it, and what expert opinion thought of the work. Editorials alerted readers to professional issues, challenges, and developments of all kinds.

Special issues maintain that tradition, and provide a reason for readers to look for a specific group of papers; done well, this can generate what might be termed "brand loyalty" in other contexts. But, as noted, it is a time-consuming strategy that depends on committed authors, timely reviewing and strong marketing. It also increasingly runs counter to the manner in which most academics view publication. For them, placing a manuscript is a function of an intuitive equation, which can be solved for the following three terms: the prestige of the journal (measured by Impact Factors or similar); the friction of the review process (expressed in terms of rejection rates); and the speed of the process (which in some fields can still extend to years). Most authors want to maximize the first term ("a couple of papers in Nature pretty much makes your career"), but understand that as it increases, the other two increase too (Copley, quoted in Phillips [5], p. 24).

In addition, for many academic authors, the activity of publication has become bifurcated. One part of it is related to scientific communication, as occurred in the past with core journals. But increasingly this has shifted to other media-prepublication, postings, blogging —all of which permit immediate interaction and minimal gatekeeping. The other part is more formal, and is linked more closely to personnel review. In many fields, it now appears that having something published in a prescribed journal is the key accomplishment, regardless of whether it is ever read or cited.

What this sums to is a fragmented market for scholarly journals, in which individuals play many roles, which do not necessarily cumulate as they once did. Authors want to be published but do not necessarily see any advantage in acting as a reviewer for a specific journal. Indeed, in a rapidly expanding marketplace, it may be hard to even differentiate all the different journals with similar names. With the advent of simple-to-use search engines such as Google Scholar, the value of going to a journal's website is reduced (and I assume that virtually no-one ever sees a print version of a journal unless it comes as part of society subscription). More crucially, with advanced sites such as Scopus, algorithms can push individual papers to readers regardless of the journals in which they are published. In this academic version of Amazon, a competent searcher can rapidly amass a collection of pdfs on a topic without ever paying any attention to journal titles.

This all adds up to a now-familiar landscape for editors, who struggle to push their "brand" and to maintain quality in the face of different author expectations, given that they are seeking to minimize the friction of getting published and care little for the academic community that a journal once represented. This is summarized rather well by Ali Modarres [6], who offers a strong critique of what is happening in the editor's chair (2015).

This raises an interesting question with regard to open access. This did not emerge as a key issue in the papers that follow, except to Steinberg [2] who takes a rote political stance on the "right to access" question. However, it is not at all clear that this is the pivotal issue to most authors. In the new marketplace, where the journal functions as a route to promotion rather than a mode of communication, its copyright policies are of relatively minor importance, as we can see from the relatively small number of authors who bother to choose OA options offered by the commercial publishers. One editor/author dramatically pulled out of this project at the last moment when he thought that he might have to pay an OA fee to have his paper published. 


\section{The Future}

What this exercise has suggested to me is that the scholarly journal has of course gone through a process of serious metamorphosis in the context of delivery, but is rapidly sowing the seeds for a second major transformation. There will always be some journals with a committed cohort of likeminded individuals, such as the network described by Hiroshi Okano [7] in his paper about the journal City, Culture and Society. Being an editor for such a journal will usually generate its own rewards of academic fellowship.

Yet for many-perhaps the bulk of those in the journal marketplace-the role of editor is becoming a more thankless role. Much of the frustration implicit in the task is demonstrated by Modarres [6], who reports on a process of speeding up at the expense of quality (Steinberg touches on this too). Peer review increasingly becomes a way to detect fraud and manage workload, a process of triage rather than a collaborative exercise in helping authors publish their very best work.

Anecdotally, this was manifested to me when trying to assemble this special issue. In contacting several dozen editors, in most instances received no response whatsoever, even when following up on a personal introduction from a third party via a university email address, and doing so two or three times. This may of course simply be poor manners, but it may also speak to the stress of being an editor, for whom another communication is simply another burden.

The long term result of this will be that becoming a journal editor will be another task that is shunned by any sensible person, somewhat akin to being a department chair or a dean; unlike these roles, it is though not well remunerated. And in small ways, we already see this occurring, with a proliferation of managing editors and other production staff taking on roles that were formally done by academics. This is no comment whatsoever on the quality of the work done by these individuals: it probably makes for a more efficient journal with fewer potential conflicts of interest. But it does imply another qualitative change within the academy, and in this we see another manifestation of the Fordist speed-up that is now so familiar. With some three quarters of academic positions held by part-time faculty, the rewards of any administrative task diminish for those few full time faculty members who have a tenured position and who see so many other claims upon their time.

\section{Conclusions}

This special issue offers a glimpse into the quotidian realities of several journal editors, which is valuable in itself. However, it also points to the complexities of the role: indeed, Modarres [6] proclaims the death of the editor. Premature as that may be, it is an open question what the future offers. While it is taken for granted in many academic circles that large commercial publishers are the enemy of good scholarly practice, any threats to their dominance do not reside in a shortage of authors, but in a looming shortage of editors who are able and willing to take on that role. 


\section{Acknowledgments}

I thank those of my colleagues who gave up their time to contribute to this project. To those of you who agreed to participate and then walked away, I would urge you to rethink your ethical standards, or at least to be more realistic in your time management practices. To those of you who did not even bother to respond: I hope this is not how you treat your authors, but I suspect it is.

\section{Conflicts of Interest}

The author declares no conflict of interest.

\section{References}

1. Kirby, A. Journal Startup in the Digital Era. Publications 2015, 3, 219-231.

2. Steinberg, P. Reclaiming Society Publishing. Publications 2015, 3, 150-154.

3. Mindell, J.S. Setting up the Journal of Transport and Health, a New Cross-Disciplinary Journal. Publications 2015, 3, 120-130.

4. Brooks, M.R.; Ison, S. Themed Volumes: A Curse or a Blessing? Publications 2015, 3, 174-189.

5. Phillips, A. Blog to the future? Journals publishing in the twenty-first century. J. Sch. Publ. 2010, 42, 16-30.

6. Modarres, A. It's not just the author: The reader and the editor are dead, too. Publications 2015, 3, 168-173.

7. Okano, H. Cultural Editing and Linking among City, Culture and Society. Publications 2015, 3, 211-218.

(C) 2015 by the author; licensee MDPI, Basel, Switzerland. This article is an open access article distributed under the terms and conditions of the Creative Commons Attribution license (http://creativecommons.org/licenses/by/4.0/). 